EPJ Web of Conferences 80, 00020 (2014)

DOI: $10.1051 /$ epjconf/20148000020

(C) Owned by the authors, published by EDP Sciences, 2014

\title{
Recent Results from the CMS Experiment at the LHC
}

\author{
Bora Isildak ${ }^{1,2, a}$ \\ ${ }^{1}$ Ozyegin University, Department of Natural and Mathematical Sciences, Nisantepe District, Orman Street, \\ 34794 Cekmekoy - ISTANBUL \\ ${ }^{2}$ The CMS Experiment
}

\begin{abstract}
Numerous studies on Jet Production, Vector Boson Production, V+Jets Production and Multi-Boson Production have been carried out by the Compact Muon Solenoid (CMS) Collaboration to test perturbative quantum chromodynamics (QCD) predictions, and to put more stringent constraints on PDFs (Parton Distribution Functions). In this paper, some of these experimental results will be presented, and their possible impacts on Higgs physics and new physics searches will be discussed.
\end{abstract}

\section{Introduction}

The LHC is the worlds largest and the most powerful collider which is $27 \mathrm{~km}$ circumference long, and has been run at $7 \mathrm{TeV}$ and $8 \mathrm{TeV}$ of pp center of mass energies so far. The collider hosts four main detectors (ALICE, ATLAS, CMS, LHCb). The first pp collisions have taken place in March 2010, the first $\mathrm{Pb}-\mathrm{Pb}$ collisions have taken in November 2010, and the first $\mathrm{p}-\mathrm{Pb}$ collisions have taken in September 2012. The Compact Muon Solenoid (CMS) is a multi purpose detector at the LHC which consists of several layers where each one is specialized to measure and identify different classes of particles. The detector requirements for the CMS along with its scientific targets can be given as; a good muon identification and momentum resolution, a good charged particle momentum resolution and reconstruction efficiency in the inner tracker, a good electromagnetic energy resolution, good diphoton and di-electron mass resolution, and good missing transverse energy (MET) and dijet mass resolution, having the word good as the maximum possible excellence with the current technology. LHC pp collisions covers an extensive and unexplored kinematic region. In these pp collisions at LHC, QCD processes are the dominant processes. They affect all measurements; as signal or as background. Hence, it must be understood in great detail. A more precise knowledge of QCD is important for new physics searches as well as for SUSY searches where the multijet production plays an important role.

\section{Higgs Discovery at CMS}

CMS Collaboration has investigated for an evidence of a Standard Model Higgs boson in five different predicted decay channels: $H \rightarrow \gamma \gamma, H \rightarrow Z Z, H \rightarrow W W, H \rightarrow \tau \tau, H \rightarrow b b$. The searches have been performed with the data samples recorded in 2011 and 2012 corresponding to integrated luminosities

abora.isildak@cern.ch 

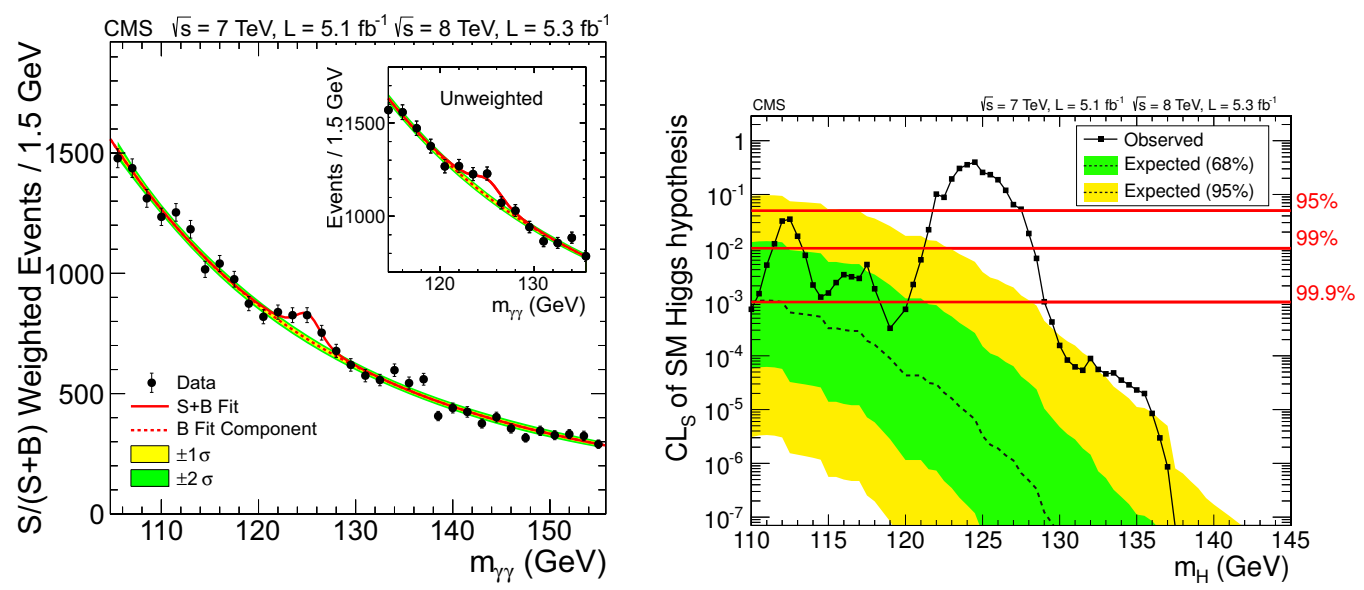

Figure 1: The diphoton invariant mass distribution with each event weighted by the $S /(S+B)$ value of its category (left). The $\mathrm{CL}_{s}$ values for the SM Higgs boson hypothesis as a function of the Higgs boson mass in the range 110-145 GeV. The background-only expectations are represented by their median (dashed line) and by the $68 \%$ and $95 \%$ CL bands (right).

of up to $5.1 \mathrm{fb}^{-1}$ at $\sqrt{s}=7 \mathrm{TeV}$ and $5.3 \mathrm{fb}^{-1}$ at $\sqrt{s}=8 \mathrm{TeV}[1]^{1}$. An excess of events is observed above the SM background which indicates the existence of a new particle. This excess has a local significance of $5.0 \sigma$, at a mass value of near $125 \mathrm{GeV}$ where the expected local significance for a SM Higgs boson of that mass is $5.8 \sigma$. The global p-value in the search range of 115-130 (110-145) GeV corresponds to $4.6 \sigma(4.5 \sigma)$. The excess is most significant in the two decay modes with the best mass resolution, $\gamma \gamma$ and $\mathrm{ZZ}$, and a fit to these signals gives a mass of $125.3 \pm 0.4$ (stat.) \pm 0.5 (syst.) $\mathrm{GeV}$. Studies on the spin and the parity of this newly discovered particle indicate that it is the foreseen scalar boson [3] .

\section{QCD Results from the CMS}

\subsection{Jet Reconstruction and Calibration}

Jets are invaluable objects to probe QCD. They are experimental signatures of quarks and gluons. A jet is an object that is clustered out of collimated spray of particles by using a set of mathematical rules. CMS's default jet reconstruction algorithm is "anti- $k_{T}$ jet" algorithm which is a successive recombination algorithm (belongs to the $k_{T}$ family). It is an infrared and collinear safe algorithm that tends to cluster around the hard energy depositions resulting geometrically cone-like jets. The jet reconstruction in CMS follows the "E-Scheme" such that addition of Lorentz vectors of massless particles ends up with massive jets. Details of the CMS jet reconstruction and calibration can be found in the related CMS publicaton [4].

\subsection{Strong Coupling Constant Measurements}

Running of the strong coupling constant $\alpha_{S}$ is a defining property of QCD physics phenomena. Hence, the determination of its evolution becomes very essential for LHC physics and for the future collider

\footnotetext{
${ }^{1} \mathrm{~A}$ recent study on the observation of the Higgs boson with $19.7 \mathrm{fb}^{-1}$ at $\sqrt{s}=8 \mathrm{TeV}$ was published after the talk was presented [2].
} 

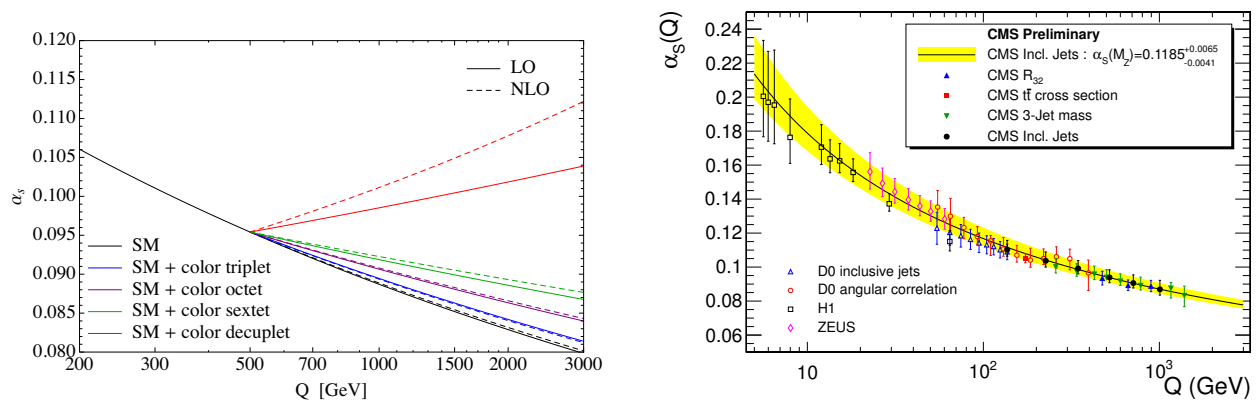

Figure 2: Change in $\alpha_{S}$ induced by a new fermion of mass $500 \mathrm{GeV}$ in various representations of the color gauge group (left)[5]. Summary of measurements of $\alpha_{S}$ as a function of the respective energy scale $Q$ [7] (right).

projects. Apart from that, new physics may affect the running of the strong coupling constant $[5,6]$. Using predictions from theory at next-to-leading order, complemented with electroweak corrections, the strong coupling constant is determined from the inclusive jet cross section to be $\alpha_{S}\left(M_{Z}\right)=0.1185$ \pm 0.0019 (exp.) \pm 0.0028 (PDF) \pm 0.0004 (NP) +0.0055 (scale), which is in agreement with the world average. All fits have also been carried out for six bins in inclusive jet $p_{T}$ separately in order to test the running of the strong coupling, where the scale $Q$ of $\alpha_{S}(Q)$ is identified with $p_{T}$. The observed behaviour of $\alpha_{S}(Q)$ is consistent with the momentum scale dependence predicted by the renormalization group equation of QCD and extend the H1, ZEUS, and D0 results to the $1 \mathrm{TeV}$ region [7].

\subsection{Dijet Mass Production Cross Section at $8 \mathrm{TeV}$}

The invariant mass of the two jets can be given in terms of proton momentum fractions $x_{1,2}$. The dijet cross section as a function of $M_{j j}$ can be precisely calculated in perturbative QCD and it also allows sensitive searches for physics beyond the Standard Model, such as dijet narrow resonances or contact interaction searches. A measurement of dijet production cross section has been performed by CMS with the data taken from LHC proton-proton collisions at $\sqrt{s}=8 \mathrm{TeV}$, corresponding to an integrated luminosty of $9.2 \mathrm{fb}^{-1}$. The spectra measured in five different rapidity bins from 0.0 to 2.5 by 0.5 intervals and the comparison with the theory predictions reported [8].

\subsection{Z Boson and b Jet(s) Cross Section}

The production of a $\mathrm{Z}$ boson, decaying into two leptons and produced in association with at least one $\mathrm{b}$ jet, is studied using $p p$ collisions in LHC at $\sqrt{s}=7 \mathrm{TeV}$. The study was done with the $5.0 \mathrm{fb}^{-1} \mathrm{of}$ data recorded in 2011 with the CMS detector. The $Z(l l)+b$-jets cross sections (where $l l=\mu \mu$ or $e e$ ) are measured for a $\mathrm{Z}$ boson produced with exactly one or at least two b jets. In addition, a cross section ratio is extracted for a $\mathrm{Z}$ boson produced with at least one $\mathrm{b}$ jet relative to a $\mathrm{Z}$ boson produced with at least one jet. The measured cross sections are compared to various theoretical predictions, and the data favor the predictions in the five-flavor $(5 \mathrm{~F})$ scheme, where b quarks are assumed massless. The kinematic properties of the reconstructed particles are compared with the predictions from the MADGRAPH event generator using the PYTHIA parton shower simulation [10]. 

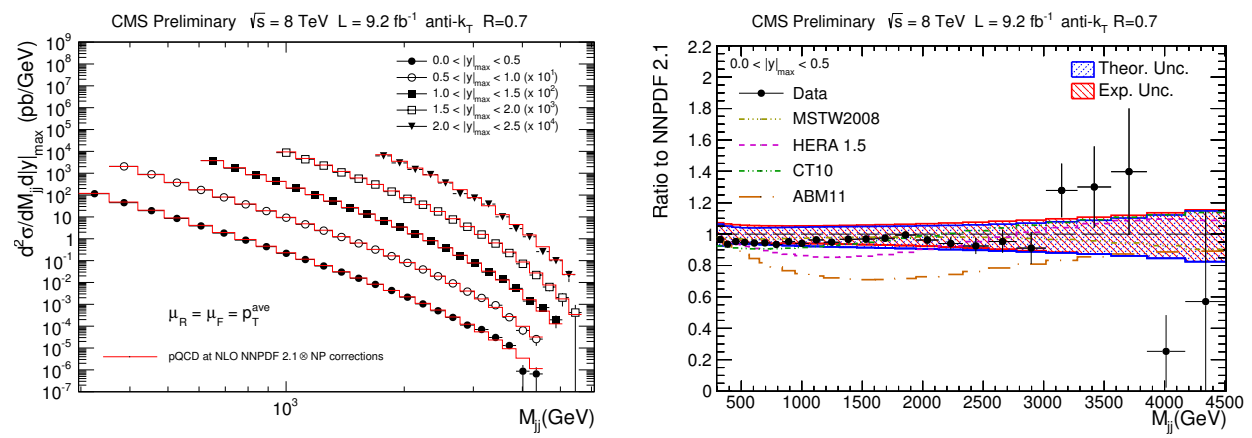

Figure 3: Dijet cross sections for the five different bins, for data (black markers) and theory (red lines) using the NNPDF2.1 PDF set (right). Summary of measurements of $\alpha_{S}$ as a function of the respective energy scale $Q[9]$ (left).

This study is of interest since it is a background in many searches for yet unobserved processes, such as the production of heavier SUSY-like Higgs bosons via vector boson fusion, and in studies of the standard model Higgs boson produced in association with a $\mathrm{Z}$ boson and decaying to $\mathrm{b}$ quark [11][12].
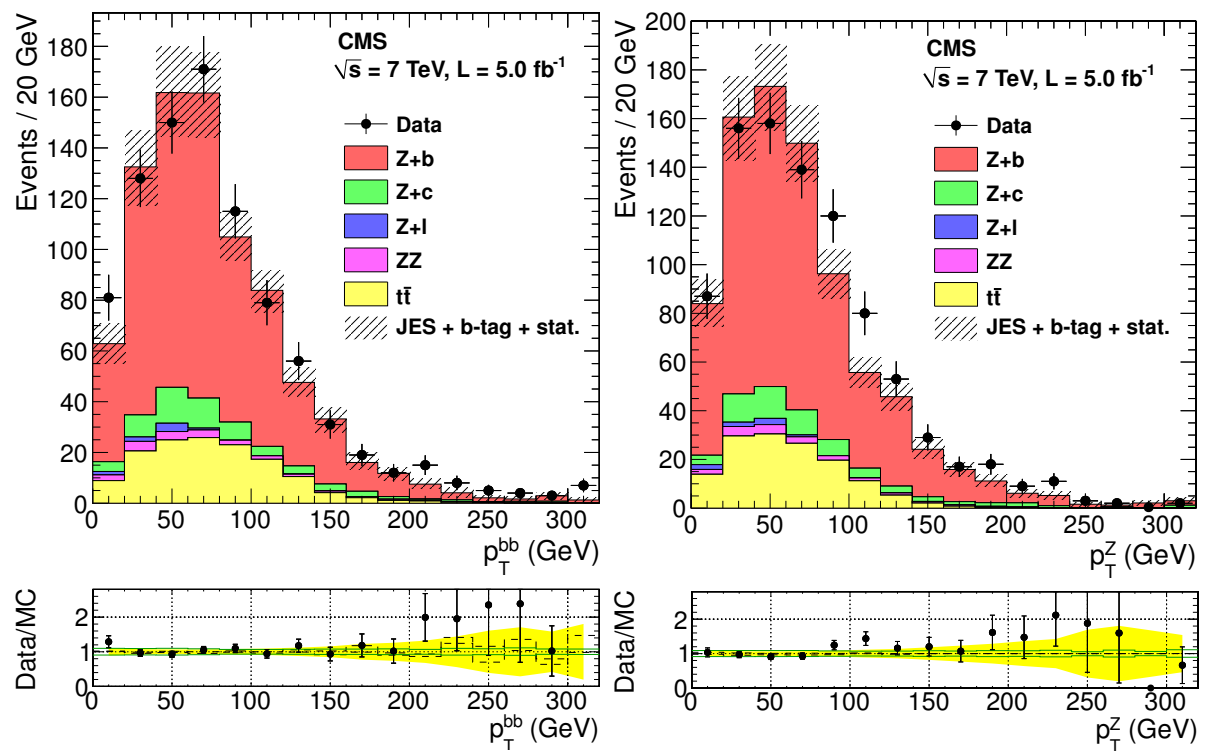

Figure 4: Distributions of kinematic observables for the $Z+2 b$-jets selection of the combined electron and muon samples, and a comparison with the simulated samples that are normalized to the theoretical predictions. First: the $p_{T}$ distribution of the dijet pair. Second: the $p_{T}$ distribution of the dilepton pair. Uncertainties in the predictions are shown as a hatched band. 
Table 1: Cross sections for the production of a $\mathrm{Z}$ boson with only one $\mathrm{b}$ jet, with at least two $\mathrm{b}$ jets, with at least one $b$ jet, and the ratio with respect to at least one jet of any flavor, showing the statistical and systematic uncertainties. The expectations from MADGRAPH, MCFM and aMC@NLO include uncertainties due to scale variations.

\begin{tabular}{l|c|ccccc}
\hline Cross section & Measured & $\begin{array}{c}\text { MADGRAPH } \\
(5 \mathrm{~F})\end{array}$ & $\begin{array}{c}\text { aMC@NLO } \\
(5 \mathrm{~F})\end{array}$ & $\begin{array}{c}\text { MCFM } \\
\text { (parton level) }\end{array}$ & $\begin{array}{c}\text { MADGRAPH } \\
(4 \mathrm{~F})\end{array}$ & $\begin{array}{c}\text { aMC@NLO } \\
(4 \mathrm{~F})\end{array}$ \\
\hline$\sigma_{\mathrm{Z}+1 \mathrm{~b}}(\mathrm{pb})$ & $3.52 \pm 0.02 \pm 0.20$ & $3.66 \pm 0.22$ & $3.70_{-0.26}^{+0.23}$ & $3.03_{-0.36}^{+0.30}$ & $3.11_{-0.81}^{+0.47}$ & $2.36_{-0.37}^{+0.47}$ \\
$\sigma_{\mathrm{Z}+2 \mathrm{~b}}(\mathrm{pb})$ & $0.36 \pm 0.01 \pm 0.07$ & $0.37 \pm 0.07$ & $0.29_{-0.04}^{+0.04}$ & $0.29_{-0.04}^{+0.04}$ & $0.38_{-0.10}^{+0.06}$ & $0.35_{-0.06}^{+0.08}$ \\
$\sigma_{\mathrm{Z}+\mathrm{b}}(\mathrm{pb})$ & $3.88 \pm 0.02 \pm 0.22$ & $4.03 \pm 0.24$ & $3.99_{-0.29}^{+0.25}$ & $3.23_{-0.40}^{+0.34}$ & $3.49_{-0.91}^{+0.52}$ & $2.71_{-0.41}^{+0.52}$ \\
\hline$\sigma_{\mathrm{Z}+\mathrm{b} / \mathrm{Z}+\mathrm{j}}(\%)$ & $5.15 \pm 0.03 \pm 0.25$ & $5.35 \pm 0.11$ & $5.38_{-0.39}^{+0.34}$ & $4.75_{-0.27}^{+0.24}$ & $4.63_{-1.21}^{+0.69}$ & $3.65_{-0.55}^{+0.70}$ \\
\hline
\end{tabular}

\section{5 $\gamma \gamma$ Differential Cross Section}

The measurement of differential $\gamma \gamma$ production cross sections provides an important test of perturbative and non-perturbative QCD. Diphoton production creates the major source of background in the $\gamma \gamma$ decay channel of the newly discovered Higgs boson [1], as well as to searches for physics beyond the standard model. New physics processes may also appear as non-resonant deviations from the predicted diphoton spectrum in events with large missing $E_{T}$. Alternatively, some models such as the graviton in the Randall-Sundrum model for warped extra dimensions even predict narrow resonances. In CMS, such cross section measurement for the production of a pair of isolated photons in $p p$ collisions has been done with $5.0 \mathrm{fb}^{-1}$ of data as an update of a previous measurement [13]. It probes a phase space defined by a highly asymmetric selection for the transverse energy $\left(E_{T}\right)$ of the two photons. This is the first time that such a phase space is probed at hadron colliders.
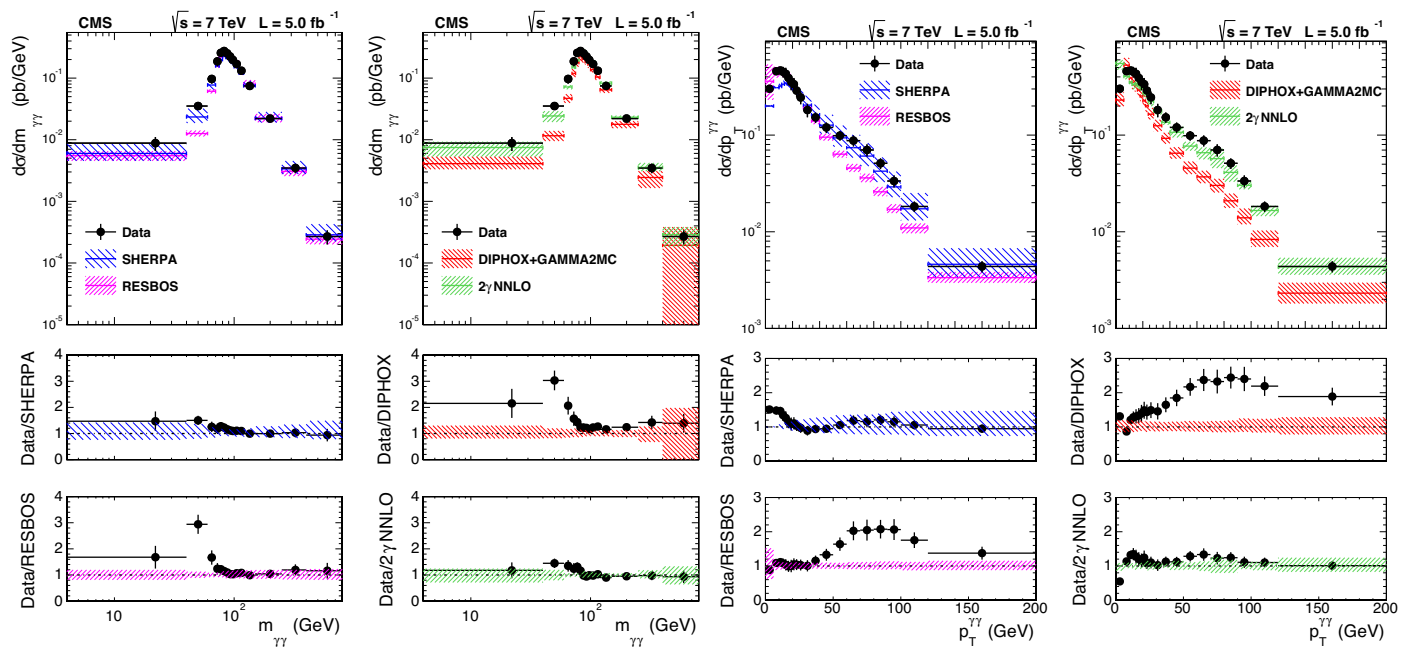

Figure 5: The comparisons of the differential cross section between data and the SHERPA, DIPHOX + GAMMA2MC, RESBOS and $2 \gamma \gamma \mathrm{NNLO}$ predictions. 


\subsection{WZ and ZZ production in final states with b-tagged jets}

Measurements are reported of the $\mathrm{WZ}$ and $\mathrm{ZZ}$ production cross sections in proton $\sqrt{s}=8 \mathrm{TeV}$ in final states where one $\mathrm{Z}$ boson decays to b-tagged jets [14]. The other gauge boson, either $\mathrm{W}$ or $\mathrm{Z}$, is detected through its leptonic decay (either $\mathrm{W} \rightarrow e v, \mu v$ or $\mathrm{Z} \rightarrow e^{+} e^{-}, \mu^{+} \mu^{-}$, or $v v$ ). The results are based on data corresponding to an integrated luminosity of $18.9 \mathrm{fb}^{-1}$ collected with the CMS detector at LHC. The measured cross sections, $\sigma(\mathrm{pp} \rightarrow \mathrm{WZ})=30.7 \pm 9.3$ (stat.) \pm 7.1 (syst.) \pm 4.1 (th.) \pm 1.0 (lum.) pb and $\sigma(\mathrm{pp} \rightarrow \mathrm{ZZ})=6.5 \pm 1.7$ (stat.) \pm 1.0 (syst.) \pm 0.9 (th.) \pm 0.2 (lum.) pb, are consistent with NLO QCD calculations.
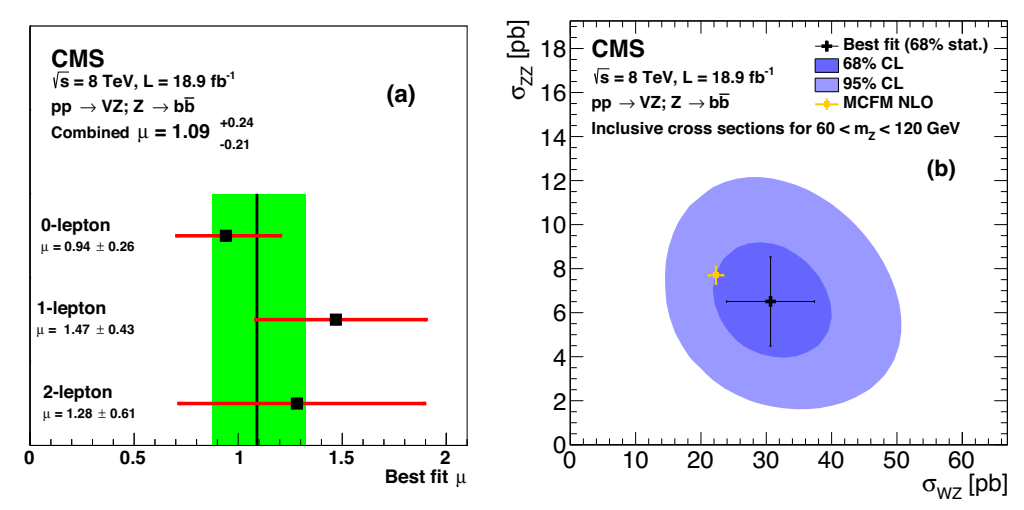

Figure 6: (a) Best-fit values of the ratios of the VZ production cross sections, relative to SM predictions for individual channels, and for all channels combined (hatched band). (b) Contours of $68 \%$ and $95 \%$ confidence level for $\mathrm{WZ}$ and $\mathrm{ZZ}$ production cross sections. The large cross indicates the best-fit value including its $68 \%$ statistical uncertainty, and the light small cross shows the result for the MCFM NLO calculation.

\section{QCD Related New Physics Searches}

\subsection{Search for Narrow Resonances in Dijet Mass Spectrum}

As it was mentioned before, new physics may show up in the dijet mass spectrum as a bump. A search for the production of new particles decaying to pairs of partons (quarks, antiquarks, or gluons), in the dijet mass spectrum in pp collisions at $\sqrt{s}=8 \mathrm{TeV}$ has been performed with the $4.0 \mathrm{fb}^{-1}$ of data collected with the CMS in 2012 [15]. No significant evidence for narrow resonance production is observed. Upper limits are set at the $95 \%$ confidence level on the production cross section of hypothetical new particles decaying to quark- quark, quark-gluon, or gluon-gluon final states (cf. Fig.7). These limits are then translated into lower limits on the masses of new resonances in specific scenarios of physics beyond the standard model. For the first time mass limits are set for the Randall-Sundrum graviton model in the dijet channel.

\subsection{Three-Jet Resonances}

Another search for three-jet hadronic resonance production in pp collisions at $\sqrt{s}=8 \mathrm{TeV}$ has been conducted with a data of $19.4 \mathrm{fb}^{-1}[16]$. The signal models explored assume R-parity-violating supersymmetric gluino pair production and have final states with either only light-flavor jets or both light- 

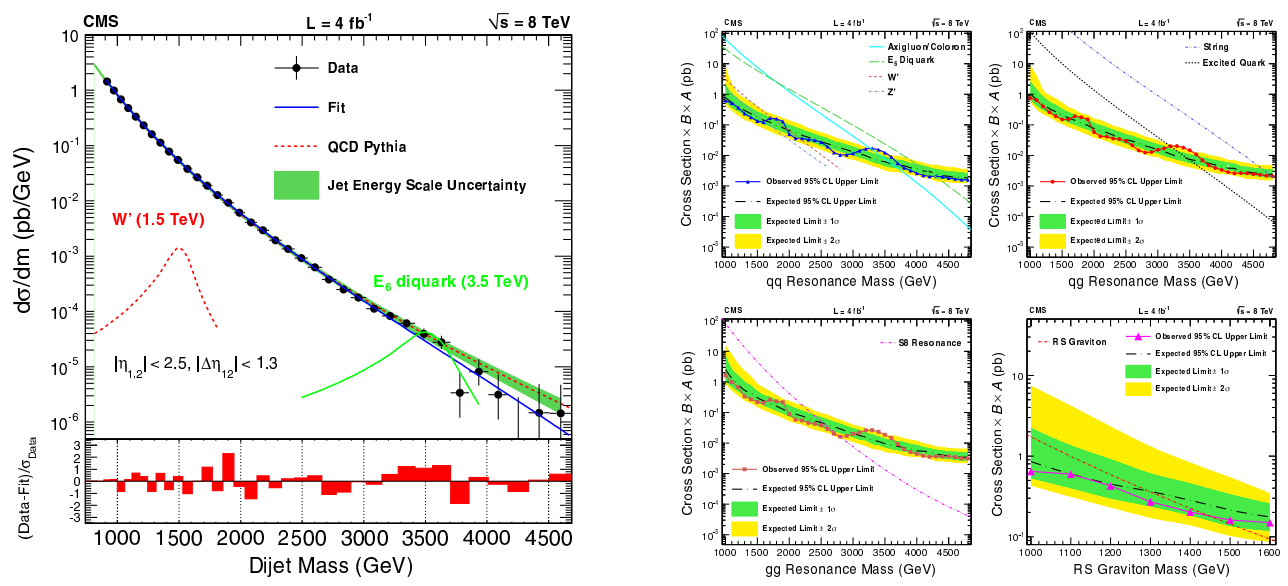

Figure 7: The dijet mass spectrum from wide jets (points) compared with a smooth fitted curve (solid line) and with the predicted QCD background (left). Observed upper limits at 95\% C.L. on $\sigma \times \mathcal{B} \times$ $\mathcal{A}$ for resonances decaying to $q q, q g$ and $g g$ final states (points and solid lines) compared with the expected limits (dot-dashed dark lines) and their variation at the $1 \sigma$ and $2 \sigma$ levels (shaded bands) (right).

and heavy-flavor jets. No significant deviation is found between the selected events and the expected standard model multijet and $t \bar{t}$ background. For a gluino decaying into light-flavor jets, a lower limit of $650 \mathrm{GeV}$ on the gluino mass is set at a $95 \%$ confidence level, and for a gluino decaying into one heavy and two light-flavor jets, gluino masses between 200 and $835 \mathrm{GeV}$ are, for the first time, excluded.
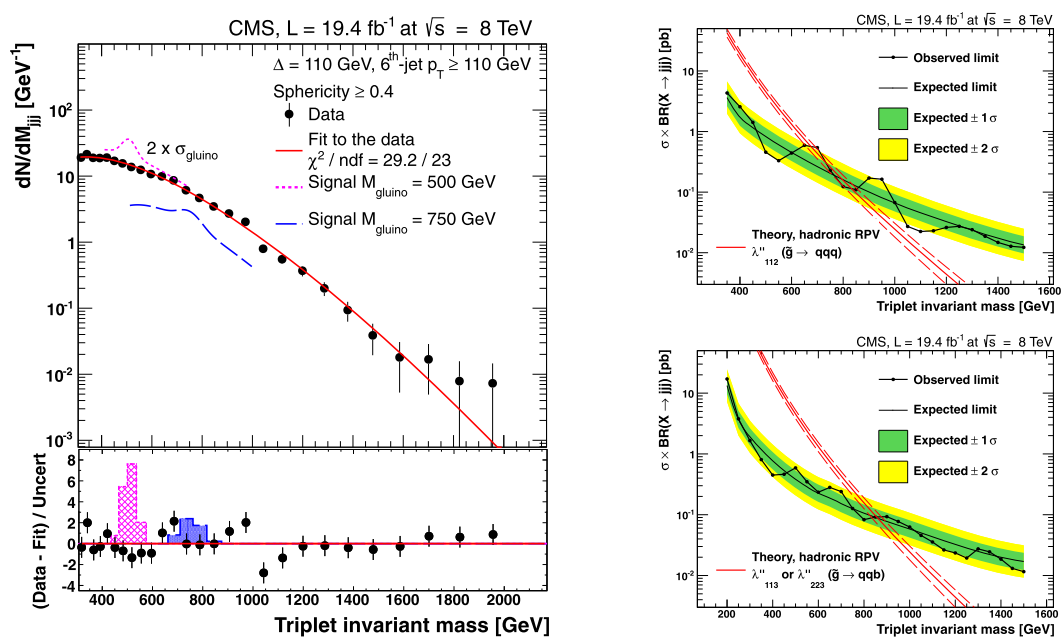

Figure 8: Comparison of the three-jet invariant mass distribution in data with the background estimate for the heavy-flavor analysis (left). Observed and expected 95\% C.L. cross section limits as a function of mass for the inclusive (top right) and heavy-flavor searches (bottom right). 


\section{References}

[1] S. Chatrchyan, V. Khachatryan, A. Sirunyan, A. Tumasyan, W. Adam, E. Aguilo, T. Bergauer, M. Dragicevic, J. Erö, C. Fabjan et al., Physics Letters B 716, 30 (2012)

[2] V. Khachatryan et al. (CMS Collaboration) (2014), arxiv . 1407.0558

[3] S. Chatrchyan, V. Khachatryan, A.M. Sirunyan, A. Tumasyan, W. Adam, E. Aguilo, T. Bergauer, M. Dragicevic, J. Erö, C. Fabjan et al. (CMS Collaboration), Phys. Rev. Lett. 110, 081803 (2013)

[4] S. Chatrchyan, V. Khachatryan, A. Sirunyan, A. Tumasyan, W. Adam, E. Aguilo, T. Bergauer, M. Dragicevic, J. Erö, C. Fabjan et al., Journal of Instrumentation 6, P11002 (2011)

[5] D. Becciolini, M. Gillioz, M. Nardecchia, F. Sannino, M. Spannowsky (2014), 1403.7411

[6] K.R. Dienes, E. Dudas, T. Gherghetta, Phys.Lett. B436, 55 (1998), hep-ph/9803466

[7] Tech. Rep. CMS-PAS-SMP-12-028, CERN, Geneva (2013)

[8] Tech. Rep. CMS-PAS-SMP-14-002, CERN, Geneva (2014)

[9] J. Beringer, J.F. Arguin, R.M. Barnett, K. Copic, O. Dahl, D.E. Groom, C.J. Lin, J. Lys, H. Murayama, C.G. Wohl et al. (Particle Data Group), Phys. Rev. D 86, 010001 (2012)

[10] S. Chatrchyan, V. Khachatryan, A. Sirunyan, A. Tumasyan, W. Adam, T. Bergauer, M. Dragicevic, J. Erö, C. Fabjan, M. Friedl et al., Journal of High Energy Physics 2014, 120 (2014)

[11] J. Campbell, R.K. Ellis, F. Maltoni, S. Willenbrock, Phys. Rev. D 73, 054007 (2006)

[12] S. Chatrchyan, V. Khachatryan, A.M. Sirunyan, A. Tumasyan, W. Adam, T. Bergauer, M. Dragicevic, J. Erö, C. Fabjan, M. Friedl et al. (CMS Collaboration), Phys. Rev. D 89, 012003 (2014)

[13] S. Chatrchyan, V. Khachatryan, A. Sirunyan, A. Tumasyan, W. Adam, T. Bergauer, M. Dragicevic, J. Erö, C. Fabjan, M. Friedl et al., Journal of High Energy Physics 2012, 133 (2012)

[14] S. Chatrchyan et al. (CMS Collaboration), Eur.Phys.J. C74, 2973 (2014), 1403. 3047

[15] S. Chatrchyan, V. Khachatryan, A.M. Sirunyan, A. Tumasyan, W. Adam, E. Aguilo, T. Bergauer, M. Dragicevic, J. Erö, C. Fabjan et al. (CMS Collaboration), Phys. Rev. D 87, 114015 (2013)

[16] tagkey2014193, Physics Letters B 730, 193 (2014) 